to the clinical setting; with senior clinicians and nursing staff being less accepting of balanced electrolyte solutions in general ward areas. Significant lack of knowledge of intravenous fluid physiology was demonstrated by some prescribers.

Conclusion Our findings demonstrate an increase in the administration of balanced electrolyte solutions, such as Plasma-Lyte 148, as fluid boluses; this is in keeping with other international surveys of fluid administration (1,2). Similarly, we have also found significant differences in fluid bolus administration practice across different clinical areas as demonstrated by Jonsson and Perner in 2017.

We have demonstrated the importance of on-going multi-disciplinary educational efforts in continuing the evolution of intravenous fluid bolus administration practice to reflect current best practice.

\section{G79(P) PHYSICIAN AND PARENTAL DECISION-MAKING PRIOR TO ACUTE MEDICAL ADMISSION}

R Barwisemunro, H Morgan, S Turner. Child Health, University of Aberdeen, Aberdeen, UK

\subsection{6/archdischild-2018-rcpch.77}

Background There is a rising number of acute medical paediatric emergency hospital admissions in England and Scotland, and the rise is explained by increased short-stay admissions. Our objective was to undertake qualitative interviews with parents and clinicians to better understand what factors, other than the health status of the child, may influence decisionmaking leading to an admission.

Methods Semi-structured interviews were conducted with parents, clinicians working in general practice, out-of-hours or accident and emergency (referring clinicians) and doctors working in acute medical paediatrics (receiving clinicians) within 24 hours of a child being admitted to hospital

Results Ten parents (including one caregiver),seven referring clinicians and ten receiving clinicians were interviewed. Parents described erring on the side of caution when seeking medical opinion and one mentioned anxiety. Among themes seen among referring clinicians, 'erring on the side of caution' was also identified as was managing 'parental anxiety' and acting on 'gut instinct'. Among receiving clinicians, themes included managing parental anxiety and increasing parental expectations of the health service.

Conclusions The study of parent and referring clinician decision-making prior to a hospital admission can identify 'teachable moments' where interventions might be delivered to slow or even arrest the rise in short-stay acute medical admissions. Interventions might, for example, assure parents or referring clinicians that hospital referral is not required and help clinicians understand what they interpret as 'parental anxiety'.

\section{G80(P) HARMONISING ANTIBIOTIC USE WITHIN THE OXFORD ACADEMIC HEALTH SCIENCES NETWORK}

${ }^{1}$ A Yannoulias, ${ }^{2}$ S Drysdale. ${ }^{1}$ Paediatrics, Frimley Health NHS Trust, Slough, UK; ${ }^{2}$ Paediatrics, John Radcliffe Hospital NHS Trust, Oxford, UK

10.1136/archdischild-2018-rcpch.78

Antibiotic resistance is a very pressing concern to the future of paediatric infections and antibiotic use. We have a duty to our patients to try and stop further antimicrobial resistance from occuring.

One way we thought we could try and tackle this very pressing concern is to establish a common guideline for antibiotic use for the common paediatric infections in the Oxford Academic Health Sciences Network (AHSN).

A total of 5 hospitals are included in the Oxford AHSN, four district general hospitals and one tertiary level hospital.This project involved a multidisciplinary team of professionals from within each hospital. The multiprofessional team included microbiology consultants, pharmacists, general paediatric consultants as well and infectious disease consultants.

Initially, in this multidisciplinary forum, the group looked at the positive microbiology culture and sensitivity results of all the sterile fluid samples sent to the laboratories, in all 5 hospitals, in the 2 years prior to the start of this project. That is looking at the years 2013-2015, within the region. The sterile fluid samples included were urine samples, blood culture samples and cerebrospinal fluid samples. This was an important intial step as we needed to know if a common guideline was plausible depending on the positive microbiology culture results. This work showed us that the organisms cultured in these samples were very similar, with similar resistance patterns within the whole region. Therefore allowing us proceed to harmonise practice of antibiotic prescribing throughout the network.

The group then looked at all the guidelines for antibiotic prescribing that were present within each hospital at the time. Looking at the similarities and differences at that time. With the results from the microbiology as stated previously and looking at the guidelines presented a consesus was reached within the group.

This project from start to finish took about 18 months to complete.

This guideline has the first line empiric antibiotic therapy recommended for the common paediatric infections including sepsis, meningitis and pneumonia. Also included in the guideline is urinary tract infections, joint infections, soft tissue infections, Ear nose and throat infections and abdominal infections.

This guideline is now embedded in practice in all of the four district general hospitals within the region as well as the tertiary level hospital.

\section{G81(P) BRIDGING THE GAP: GP HUB CLINICS AND MDT TEACHING SESSIONS}

S Suri, D Patel, S Cooper, M Simmonds. Children and Young People's Services, The Rotherham NHS Foundation Trust, Rotherham, UK

10.1136/archdischild-2018-rcpch.79

Aims Despite the fact that children account for $20 \%-25 \%$ of the caseload of GPs, training in Paediatrics is not compulsory. Public expectation is that parents should have access to specialist care immediately. Several studies have concluded that substantial numbers of children referred to secondary care can be successfully managed in primary care. These were the main drivers for setting up a GP hub clinic and Multi-disciplinary team (MDT) teaching sessions in our area.

Methods GP hub clinics were set up and evaluated over a period of 7 months between February and October 2017. Each clinic had capacity to see 5 new patients and took place 